advances in bronchoscopy, bronchial brush biopsy, and cytological examination of sputum may provide a positive diagnosis of tumour in a small proportion of these cases, negative findings do not exclude that diagnosis and should not be allowed to influence the decision on whether or not to advise resection of the lesion.

Some patients may be unfit for surgical treatment because of poor respiratory function, but there is no consensus of view on how they should be managed. Radiotherapists are often reluctant to irradiate a pulmonary lesion when there are no substantial grounds for a diagnosis of bronchial carcinoma, but will usually be prepared to do so if the opacity definitely increases in size after a period of observation. Since tuberculosis is the most likely alternative diagnosis in these cases many physicians advise antituberculosis chemotherapy during this period if the patient is a positive tuberculin reactor, on the assumption that there is nothing to be lost by "treating the treatable." Reduction in the size of the lesion after three or four months of treatment gives indirect confirmation of the diagnosis. Sputum or laryngeal swabs should have been examined for Mycobacterium tuberculosis in every case as part of the primary investigation. Another approach sometimes advocated is to give a test dose of $x$-ray therapy to the lesion. If it diminishes rapidly in size a diagnosis of bronchial carcinoma may be assumed, and a full course of treatment should then be given.

In younger patients solitary pulmonary nodules are more likely to be benign than malignant; and this might be expected to alter the approach to their management, but in practice many of these lesions are still being resected unless they are calcified. In a recent study by Trunk et al..$^{1}$ of 137 patients who had solitary pulmonary nodules resected, there were 44 patients under the age of 35 , and in none of these cases was the lesion a bronchial carcinoma. In a review of the literature Trunk et al. found that less than $1 \%$ of all resected solitary nodules in this age group were malignant, and they concluded that operation was unnecessary in such cases. On the other hand, thoracotomy is not a hazardous procedure in this age group, and resection of the lesion can provide, at little risk to the patient, the welcome security of a definite diagnosis, which in most cases will eliminate the need for a long period of regular observation. Many of these patients will also be spared unnecessary antituberculosis chemotherapy-often advised if the tuberculin test is positive. No rational policy can be formed on the management of individual patients in the face of these conflicting philosophies, and until better methods of diagnosis are available there will always be a difference of opinion on whether or not to resect solitary pulmonary nodules.

Virtually all lesions in which deposits of calcium can be demonstrated are benign and should not be resected. Calcification may be difficult to detect on conventional $x$-ray films, and the investigation of solitary pulmonary opacities should always include tomography. The differential diagnosis of a calcified opacity is mainly between tuberculosis and hamartoma, and the only problem in these cases is to decide whether or not to advise antituberculosis chemotherapy. In general, this should be given if the patient is a positive tuberculin reactor and if a substantial portion of the opacity is uncalcified, but even in these cases it may be justifiable, particularly if the lesion has a sharply defined margin and is thus more likely to be a hamartoma, to withhold treatment provided the patient can be kept under observation.

Many patients who present with solitary pulmonary opacities have been $x$-rayed before, and re-examination of the earlier films, if they are available, may confirm that the lesion has been present for long enough to exclude a diagnosis of bronchial carcinoma. It that way a useful number of unnecessary thoracotomies can be avoided.

1 Trunk, G., Gracey, D. R., and Byrd, R. B., Chest, 1974, 66, 236.

\section{Spinal Manipulation}

A perennial area of conflict between orthodox and fringe medicine lies in the place of manipulative therapy for spinal pain. The British Association for Rheumatology and Rehabilitation, with the support of the D.H.S.S., recently organized a multicentre controlled trial of the effects of manipulation on low back pain, and the results appear at p. 161 .

Any investigation of spinal pain is beset by the difficulty of finding objective, measurable features. The main symptom is pain, and the signs of limited movement and limited prone and supine leg sizetching are largely secondary to pain. Patients were admitted to the trial only if they were aged 20-50, had painful limitation of movement of the lumbar spine, and had no contraindication to any of the four treatments compared. It could be argued that it would have been better to limit the trial to two groups: one to be manipulated (mobilized) and one to be corseted (immobilized). The addition of two more groups, one treated by physiotherapy of choice and the other by analgesic tablets, contributed little and may even have blurred the focus on the early response to manipulation by halving the numbers available for analysis. Since manipulators from several "schools" participated, including an osteopathic physician, it was not possible to standardize the techniques used.

The results hinted that manipulative treatment may give quicker relief than the other treatments used, but by three weeks there were no marked differences. Glover ${ }^{1}$ reported a similar finding in a single-blind trial comparing manipulation with detuned shortwave in 81 patients : more of the manipulated patients achieved immediate relief, but this difference was no longer apparent at the end of a few days.

The results bear out the clinical impression that low back pain is a recurrent syndrome with periods of exacerbation which may last up to eight weeks but which tend to settle spontaneously. Undoubtedly some patients can be dramatically relieved by manipulation, as anyone who has experienced this personally will bear out, but such cases are difficult to preselect. Further studies should, perhaps, be designed specifically to assess the benefit of manipulation within the first week of lumbago with recent onset. There is no indication that in the long term manipulation affects the outcome either favourably or adversely. Since for the forseeable future facilities in the hospital service will have to be rationed no case has been shown for providing widespread facilities for manipulation.

Glover, J. R., Transactions of the Society of Occupational Medicine, 1971, 21,

\section{Fetal Damage from Breech Birth}

Trauma, often associated with asphyxia, is the main threat to the life of the baby in breech birth, though other factors such as low birth weight, placenta praevia, and congenital anomalies 